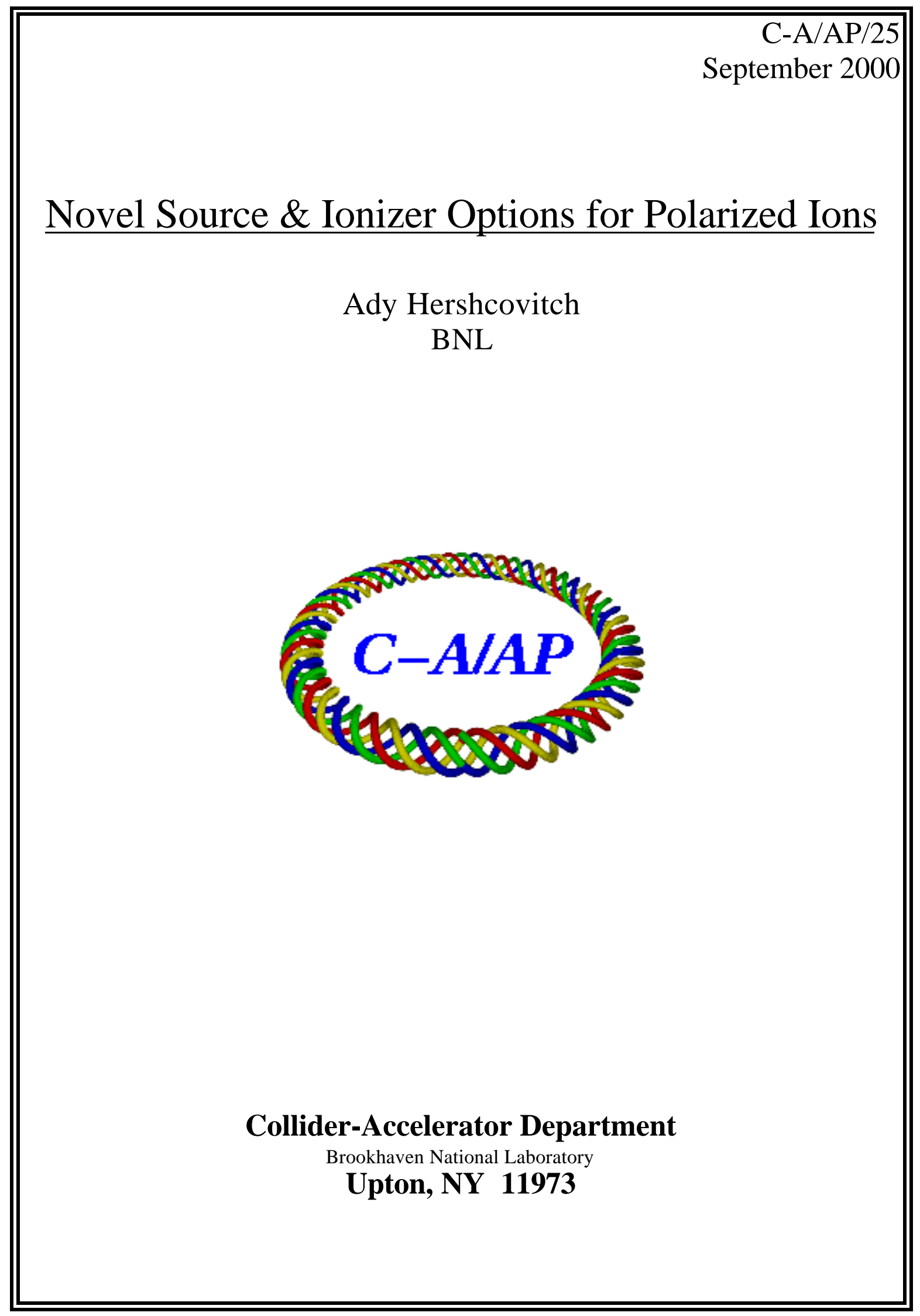




\title{
Novel Source \& Ionizer Options for Polarized Ions
}

\author{
Ady Hershcovitch
}

\begin{abstract}
Presently, the RHIC Spin project includes a polarized proton program. And, there is a future need for sources of polarized ${ }_{3} \mathrm{He}$ ions for RHIC and elsewhere. Ideas for intense sources of $\mathrm{H}^{+}$and ${ }_{3} \mathrm{He}^{++}$ions, as well as ionizer options for polarized ${ }_{3} \mathrm{He}$ ion sources are discussed. Polarized ${ }_{3} \mathrm{He}$ ions had been accelerated over thirty years ago. However, only a few ${ }_{3} \mathrm{He}$ ion sources have been in operation, even though polarized ${ }_{3} \mathrm{He}$ ion beams can be very useful in nuclear and high energy physics research. A major problem is the low output current yields and/or low polarization of these sources. A major culprit for the low outputs has been poor ionizer performance. Two types of ionizers are considered: magnetized Z-pinches and fiber cathode electron beam ionizers. Recently copious amounts of fully striped helium have been extracted from LIZ-MeV (A magnetized Zpinch ion source). Several kA of current were extracted. Discharge pulse length was 2.2 ì sec. To realize the magnitude of this output, $1 \mathrm{kA}$ of $\mathrm{He}^{++}$in this pulse length yields about $1.4 \times 10^{16} \mathrm{He}^{++}$per pulse. A minute fraction of this output would satisfy any RHIC polarized neutron program requirements. A magnetized Z-pinch ion source can generate even larger quantities of $\mathrm{H}^{+}$to enhance OPPIS. Polarized ${ }_{3} \mathrm{He}$ ion source ionizers are evaluated: one utilizes a fiber tip electron beam to ionize helium of an existing polarized ${ }_{3} \mathrm{He}$ internal target. The other method relies on fast stripping of ${ }_{3} \mathrm{He}$ atoms to form ${ }_{3} \mathrm{He}^{++}$ inside LIZ-MeV. Quick ionization (before any depolarization or other losses can occur) and simplicity are the main advantages of these approaches.
\end{abstract}

\section{INTRODUCTION}

Presently, the RHIC Spin project includes a polarized proton program, and there is interest in starting a polarized neutron program. To meet polarized proton program requirements, an Optically Pumped Polarized Ion Source (OPPIS) is being installed and commissioned at BNL. Originally built at KEK this ion source was greatly upgraded at TRIUMF. ${ }^{1}$ For neutron spin studies, a source of polarized ${ }_{3} \mathrm{He}$ ions is needed (since the spin of ${ }_{3} \mathrm{He}$ is due to its neutron).

OPPIS has generated $1.5 \mathrm{~mA}$ of polarized $\mathrm{H}$ with pulse duration of up to $600 \mu \mathrm{sec}$, and future upgrades are planed. Presently, OPPIS consists of an ECR cavity, which generates $3.5 \mathrm{keV} \mathrm{H}^{+}$ions, followed by an optically polarized $\mathrm{Rb}$ vapor cell. The protons pick up a polarized electron (for electron polarization), and drift through a Sona field (to attain nuclear polarization) into a $\mathrm{Na}$ jet vapor cell, where some of the atoms pick up an electron to become polarized $\mathrm{H}^{-}$. The $\mathrm{Rb}$ vapor cell is enclosed in a $2.5 \mathrm{~T}$ superconducting solenoid. In its fringe field of about $10 \mathrm{kG}$ is the ECR cavity, which operates with frequencies ranging from 18 to $28 \mathrm{GHz}$. Moving the solenoid varies the ECR magnetic field. Presently, the ECR generates $\mathrm{H}^{+}$ion beams with a current of $200-300$ $\mathrm{mA}$. Plans are to use a neutral beam injector from BINP and a helium cell stripper as the OPPIS upgrade. 
Polarized ${ }_{3} \mathrm{He}$ ions had been accelerated over thirty years ago. ${ }^{2,3,4}$ However, only a few ${ }_{3} \mathrm{He}$ ion sources have been in operation, even though polarized ${ }_{3} \mathrm{He}$ ion beams can be very useful in nuclear and high energy physics research. A major problem is the low output current yields and/or low polarization of these sources.

Two basic types of polarized ${ }_{3} \mathrm{He}$ ion sources have been pursued. The first type depends on preferential ionization of excite (metastable $2 \mathrm{~S}$ ) polarized ${ }_{3} \mathrm{He}$ ions or atoms. ${ }^{3,4}$ Electron polarized ${ }_{3} \mathrm{He}(2 \mathrm{~S})$ or ${ }_{3} \mathrm{He}^{+}(2 \mathrm{~S})$ are generated by either Stern - Gerlach selection or by collisonal (rf discharge) excitation and optical pumping. Polarization transfer to the nucleus follows that step. Finally, the polarized ${ }_{3} \mathrm{He}(2 \mathrm{~S})$ are ionized to form polarized ${ }_{3} \mathrm{He}^{++}$or the polarized ${ }_{3} \mathrm{He}^{+}(2 \mathrm{~S})$ are further ionized to form polarized ${ }_{3} \mathrm{He}^{++}$. Ionization is performed by electron bombardment in various discharge devices. The underline principle is that $2 \mathrm{~S}$ excited (and polarized) atoms or ions are preferentially ionized compared to the non-excited background.

In the second type, ${ }_{3} \mathrm{He}^{++}$are injected into an optically polarized $\mathrm{Rb}$ vapor cell to form electron polarized ${ }_{3} \mathrm{He}^{+}$, which is then separated from unpolarized ${ }_{3} \mathrm{He}^{++}$. Electron polarization transfer to the nucleus is the next step. Depending on the accelerator system, further stripping to ${ }_{3} \mathrm{He}^{++}$is the final step. Claims ${ }^{5}$ are, however, that electron polarized ${ }_{3} \mathrm{He}^{+}$does not survive depolarization unless it remains in an ultra high magnetic filed.

ECR, hot filament, and coil rf discharges have been used for ionization and excitation. There is a large electron energy distribution, as well as, large components of ions and atoms excited to various states in these discharges. Consequently, polarized sources, of the first type, have low output current yields and/or low polarization. The major shortcomings of the second type source is the use of an ECR source to generated a ${ }_{3} \mathrm{He}^{++}$ beam. Compared to the ionizers to be discussed in this note, the above ionizers have a low ion fraction. And, the ratio of ${ }_{3} \mathrm{He}^{++}$to singly ionized helium is $1 \%$ or less.

In this note, intense sources of $\mathrm{H}^{+}$and ${ }_{3} \mathrm{He}^{++}$ions are described, as well as ionizer options for polarized ${ }_{3} \mathrm{He}$ ion sources. One the ideas, for enhancement of polarized $\mathrm{H}$, can be explore relatively easy with the existing OPPIS facility.

\section{MAGNETIZED Z-DISCHARGE ION SOURCE}

A Z-pinch involves a sudden compression of low-density plasma by means of a large discharge current that can last for a few microseconds. Its fill pressure is below a milliTorr. First, a low-density, low-temperature plasma is created by rf or exploding wires. Second, a large voltage is applied to the end plates that drives a very large axial current that compresses the plasma due to an inward acceleration of a surface current shell. Discharge currents of 10 MA over a few centimeters have been exceeded in a rather expensive premier device at SNL. ${ }^{6}$ This machine is one of the options being followed in an ongoing effort to attain controlled thermonuclear fusion. To improved stability, magnetized pinches were introduced in the 1950's. In a series of experiments with magnetized (axial magnetic field of 1.5 T) Z-pinches, $2 \mathrm{MA}$ were reached for a length of 
0.8 meters with a pulse length of 250 microseconds. This approach was abandoned, since the maximum ion temperature reached was only $50 \mathrm{eV} .^{7}$

Low ion temperature plasmas, coupled with very high currents (ion contribution to the total discharge current can reach $10 \%$ ), are ideally suited for intense ion sources. Thus, magnetized Z-pinches can be excellent pulsed ion sources. Although a $10 \mathrm{MA}$ Z-pinch is a very large device, tabletop systems (no larger than the ECR) can handle $100 \mathrm{kA}$. Magnetized Z-discharges can have hollow structural geometry like the LIZ-MeV device at UCI. This particular device is used to generate heavy metallic ions. Recent experiments indicate that the extracted ion current is in the range of kilo-Amperes. In addition to the very large current yield, a Z-discharge based $\mathrm{H}^{+}$ion source will have a number of additional advantages.

This ion source has a hollow geometry and it operates well in any (or no) magnetic field. There is no need to match resonance. The present ECR source is in fringing magnetic field that varies throughout an ECR, hence, the resonance region is small. Unlike the BINP injector and stripper, Z-discharge can be placed adjacent to $\mathrm{Rb}$ cell. Its hollow geometry is well suitable for allowing laser light into the $\mathrm{Rb}$ cell.

Recently copious amounts of fully striped helium have been extracted from LIZ-MeV. Several kA of current were extracted. Discharge pulse length was 2.2 ì sec. To realize the magnitude of this output, $1 \mathrm{kA}$ of $\mathrm{He}^{++}$in this pulse length yields about $1.4 \times 10^{16} \mathrm{He}^{++}$ ions per pulse. A minute fraction of this output would satisfy any RHIC polarized neutron program requirements.

\section{FIBER TIP CATHODE ELECTRON BEAM IONIZER}

Electron beams can be rather effective strippers. The polarized ion source at the Saturne National Laboratory in, SACLAY France, ${ }^{8}$ and the E-Mevva heavy ion source are good examples where an electron beams have been used as strippers. For effective stripping, atoms must be subjected to bombardment by a sufficient number of streaming electrons. This number is basically the product of atom exposure time to the beam and the electron beam density. Large research programs in inertial confinement fusion and in the intense microwave tube industry spearheaded the development of powerful electron beams. Mega-Ampere electron beams have been generated by diodes. Although most of these diodes operate with pulses that are in the nsec range, some diodes have operated with pulse lengths of up to 2 microseconds. These powerful diodes require large systems to drive them. In any case such a beam is overkill.

Fiber tip cathodes are, basically, field emission cathodes. Commercially available carbon fibers have typical diameters of $5: \mathrm{m}$ (Thornel ${ }^{\circledR}$ ) or $7: \mathrm{m}$ (Carborundum Co.). As a consequence of their microscopic size, applied moderate voltage result in very high electric fields at the tips. Record current density yields exceeding $10^{7} \mathrm{~A} / \mathrm{cm}^{2}$ have been obtained with a $0.1: \mathrm{m}$ tungsten tip cathode. 


\section{APPLICATIONS}

Next, possible utilization of these ionizers in various polarized ion sources is described.

\section{A. OPPIS}

Presently, an ECR ion source generates $3.5 \mathrm{keV} \mathrm{H}^{+}$ions for injection into the optically polarized Rb vapor cell of OPPIS. This ECR has a current output of $200-300 \mathrm{~mA}$. Improving OPPIS may be possible with a magnetized Z-discharge, like LIZ-MeV, to feed $\mathrm{H}^{+}$into the optically pumped $\mathrm{Rb}$ cell (instead of ECR source).

Given the recent performance by LIZ-MeV, a similar ion source generating at least $10 \mathrm{~A}$ of $\mathrm{H}^{+}$in pulses of $150: \mathrm{sec}$ duration is achievable. This source would yield $9.4 \times 10^{15} \mathrm{H}^{+}$ per pulse. This output is orders of magnitude higher than the RHIC program requirements. Therefore, the source can be scaled down if its engineering problems become too challenging. In addition to enhanced $\mathrm{H}^{+}$output, the source has additional advantages. It can use the magnetic field coil of the $\mathrm{Rb}$ vapor cell regardless of its location, since there is no need to match rf resonance. Due to its $100 \%$ ionization there is a reduced gas problem.

A description of LIZ-MeV can be found elsewhere. Figure 1 shows a schematic of LIZ$\mathrm{MeV}$.

\section{B. $\quad$ Polarized ${ }_{3} H e$ Ion Sources}

LIZ-MeV and the electron beam stripper are evaluated as ionizers for polarized ${ }_{3} \mathrm{He}$ ion sources. First a polarized ${ }_{3} \mathrm{He}$ gas is generated and introduced into an ionizer, followed by fast stripping of the gas to form polarized ${ }_{3} \mathrm{He}^{++}$ions.

Two major mechanisms have been employed to polarized ${ }_{3} \mathrm{He}$ gas: direct optical pumping, and spin-exchange collisions. Direct optical pumping is performed on excited (metastable $2 \mathrm{~S}$ ) of ${ }_{3} \mathrm{He}$ atoms. Spin-exchange collisions take place in a polarized (optically pumped) alkali-metal vapor cell. Next step is to ionize the polarized ${ }_{3} \mathrm{He}$ with an electron beam. An internal target setup can adapted for this task.

For several years, the polarized ion source at Saturne National Laboratory in, SACLAY France, had generated ion beams of highly polarized hydrogen and deuterium ions with currents exceeding $700 \mu \mathrm{A}$. Just prior to the closure of Saturne, polarized ion currents of $2.5 \mathrm{~mA}$ had been reached. The SACLAY source was an ABS with an electron beam ionizer. Improvements of the electron beam ionizer lead to the large increase in output (from 0.7 to $2.5 \mathrm{~mA}$ ). ${ }^{8}$ Fiber tip can generate much larger currents (and current densities) than conventional electron beam ionizers.

Fiber tip cathode can easily generate a $5 \mathrm{kA}$ electron beam with a current density of 5 $\mathrm{kA} / \mathrm{cm}^{2}$. It can operate in large magnetic field, e.g., the same solenoid as the OPPIS optically pumped cell. Or, the old BNL cold atomic beam solenoid may be used. The ionizer can be rather short. Ionization to ${ }_{3} \mathrm{He}^{+}$occurs in less than one microsecond. By 
comparison, it takes a $300 \mathrm{~K}$ (room temperature) He $12.8: \mathrm{sec}$ to move $1 \mathrm{~cm}$. Ionization time $\mathrm{J}_{\mathrm{i}}=\left(\mathrm{nF} \mathrm{v}^{-1}\right.$, where $\mathrm{F}$ in the ionization cross section, $\mathrm{n}$ and $\mathrm{v}$ are electron beam density and velocity respectively. Given a current density $j, n v=j / 1.6 \times 10^{-19}$. For $\mathrm{He}+\mathrm{e} \rightarrow \mathrm{He}^{+}, \mathrm{F}=3.8 \times 10^{-17} \mathrm{~cm}^{2}$, therefore, $\mathrm{J}_{\mathrm{i}}=(\mathrm{nFv})^{-1}=842 \mathrm{nsec}<1:$ sec. If there is need for ${ }_{3} \mathrm{He}^{++}$polarized, $\mathrm{F}=5 \times 10^{-18} \mathrm{~cm}^{2}$ for $\mathrm{He}^{+}+\mathrm{e} \rightarrow \mathrm{He}^{++}$, hence $\mathrm{J}_{\mathrm{i}}=6.4: \mathrm{sec}$ (full stripping in about $0.5 \mathrm{~cm}$ ). The desired number of ions per pulse would determine ionizer length. A $5 \mathrm{kA} @ 10 \mathrm{kV} 1 \mathrm{~m}$ long ionizer has a capacity of $4.8 \times 10^{14}$ charges (polarized helium ions).

A schematic of a polarized ${ }_{3} \mathrm{He}^{++}$ion source, based on an electron beam ionizer, is shown in figure 2. The configuration is a variation on a an internal polarized ${ }_{3} \mathrm{He}$ gas target. Instead of a beam line, the polarized ${ }_{3} \mathrm{He}$ gas is to be subjected to ionization by an intense electron beam in a strong magnetic field. Fiber-tip cathodes generate the electron beam currents.

Depolarization effect of an electron beam ionizer is an issue that must be addressed. The electron beam current generates an azimuthal magnetic field given by $\mathrm{B}=0.2 \mathrm{I} / \mathrm{r}$, where $\mathrm{I}$ is the current in Amperes and $\mathrm{r}$ is the radial location in centimeters. At the electron beam perimeter, $\mathrm{B}=1772$ Gauss. The precession period of ${ }_{3} \mathrm{He}$ in this magnetic field is 1.2 microseconds (given by $\mathrm{T}^{-1}$, where $\mathrm{T}=\mathrm{g}:{ }_{n} \mathrm{~B} / \mathrm{h}, \mathrm{:}_{\mathrm{n}}$ is the nuclear magnetron). For a neutron $\mathrm{g}=1.9$, hence $\mathrm{T}=\mathrm{g}:{ }_{\mathrm{n}} \mathrm{B} / \mathrm{h}=8.5 \times 10^{5} \mathrm{~Hz}$. However, if a strong axial magnetic field exceeding 6 tesla is maintained during the ionization process, no depolarization problems are expected.

An alternative solution, to the ultra high magnetic field, is to ionize the polarized ${ }_{3} \mathrm{He}$ gas with contrastreaming electron beams in a lower magnetic field. A second (hollow) fibertip cathode is to be placed if front of the extractor in figure 2. In this configuration, the azimuthal magnetic field, and hence, the depolarization source is eliminated. To maintain the same ionization time and trap capacity, $2.5 \mathrm{kA} @ 2.5 \mathrm{kA} / \mathrm{cm}^{2}$, per cathode, are needed. Reducing the extracted current density in each cathode allows for reduction in electron gun extraction voltage, and hence reduction in trap length. At these parameters, no beam instabilities will occur.

\section{B2. Capture of polarized electrons}

An alternative method is based on ${ }_{3} \mathrm{He}^{++} \Rightarrow{ }_{3} \mathrm{He}^{+}$in a polarized $\mathrm{Rb}$ vapor cell. In this method, ${ }_{3} \mathrm{He}^{++}$ions are injected into an optically polarized $\mathrm{Rb}$ vapor cell to form electron polarized ${ }_{3} \mathrm{He}^{+}$, which is then separated [with a velocity selector, e.g., Wein filter (ExB analyzer)] from the unpolarized ${ }_{3} \mathrm{He}^{++}$. Electron polarization transfer to the nucleus, by subsequent hyperfine interactions, is the next step. The following step is to ionize ${ }_{3} \mathrm{He}^{+}$to ${ }_{3} \mathrm{He}^{++}$with an electron beam ionizer or even a second LIZ-MeV.

Recent output from LIZ-MeV is about $1.4 \times 10^{16} \mathrm{He}^{++}$ions per pulse. In a previous study, $\mathrm{He}^{++}+\mathrm{e} \rightarrow \mathrm{He}^{+}$had $6 \%$ efficiency in a polarized sodium vapor cell with target thickness of about $5 \times 10^{13} \mathrm{~cm}^{-2}$. With $6 \%$, efficiency LIZ-MeV can $8.4 \times 10^{14}$ polarized ${ }_{3} \mathrm{He}^{+}$per pulse. With a $10 \%$ ionization efficiency, $8.4 \times 10^{13}$ polarized ${ }_{3} \mathrm{He}^{++}$per pulse can be obtained. 
Even a small fraction of this output would satisfy any RHIC polarized neutron program requirements. And yet, the OPPIS $\mathrm{Rb}$ cell has a larger target thickness, and a fiber-tip electron beam ionizer has an efficiency exceed an ionization efficiency of $10 \%$. To prevent depolarization, an axial magnetic field of 6 tesla must be applied to LIZ-MeV, the OPPIS Rb cell, and to the ionizer.

\section{DISCUSSION}

A modified LIZ-MeV could generate copious amounts of either $\mathrm{H}^{+}$or $\mathrm{He}^{++}$. Huge $\mathrm{He}^{++}$ output implies that even poor efficiencies in the other steps needed to generate polarized $\mathrm{He}^{++}$would produce sufficient quantities of polarized $\mathrm{He}^{++}$. Presently, LIZ-MeV is designed to generate high charge state heavy ions, requiring discharges characterized by high current and high voltage. Hence, there is a need to cool this pulse source. But, source arc voltage (power) can be scaled down if its engineering problems become too challenging. Unlike high a charge state heavy ion source that requires energetic electrons, the second ionization potential for helium is $54.2 \mathrm{~V}$. Furthermore, the cross-section for the reaction $\mathrm{He}^{+}+\mathrm{e} \rightarrow \mathrm{He}^{++}$peaks at $175 \mathrm{eV}$ (an order of magnitude lower than the LIZ$\mathrm{MeV}$ discharge voltage).

Schemes based on LIZ-MeV and the fiber tip cathode electron beam ionizer are compatible with the OPPIS setup due to their hollow geometry. Most of the additional components are inexpensive and are relatively simple. For polarized ${ }_{3} \mathrm{He}$ be generated in a large magnetic field of 5 tesla or higher would be needed. Proof-of-principle experiments can be performed during periods, in which OPPIS is idle.

\section{References}

1). C.D.P. Levy and A.N. Zelenski, Rev. Sci. Instrum. 69, 732 (1998).

2). S. Baker, E. Carter, D. Findley, L. Hatfeld, G. Phillips, N. Stockwell, and G. Walters, Phys. Rev. Lett. 20, 738 (1968).

3). W. Burcham, O. Karban, S. Oh, and W. Powell NIM 116, 2 (1974).

4). R. Slobodrian, C. Rioux, J. Giroux, and R. Roy, AIP Conf. Proc. \# 131, 8 (1985).

5). A.N. Zelenski, private communication (2000).

6). Work by McDaniel, et al., D.L. Cook, private communication, 1994; Rahman, Wessel, and Rostoker, reported current levels of 5 MA in PRL 74, 714 (1995).

7). A series of papers (by Golovin, et al., Komelkov, et al., and Andrianov, et al.), presented at the second U.N. Conf. on Peaceful Uses of Atomic Energy, Vol. 31 and 32, Geneva, 1958.

8). J-L Lemaire, AIP Conf. Proc. \# 293, 106 (1993). 


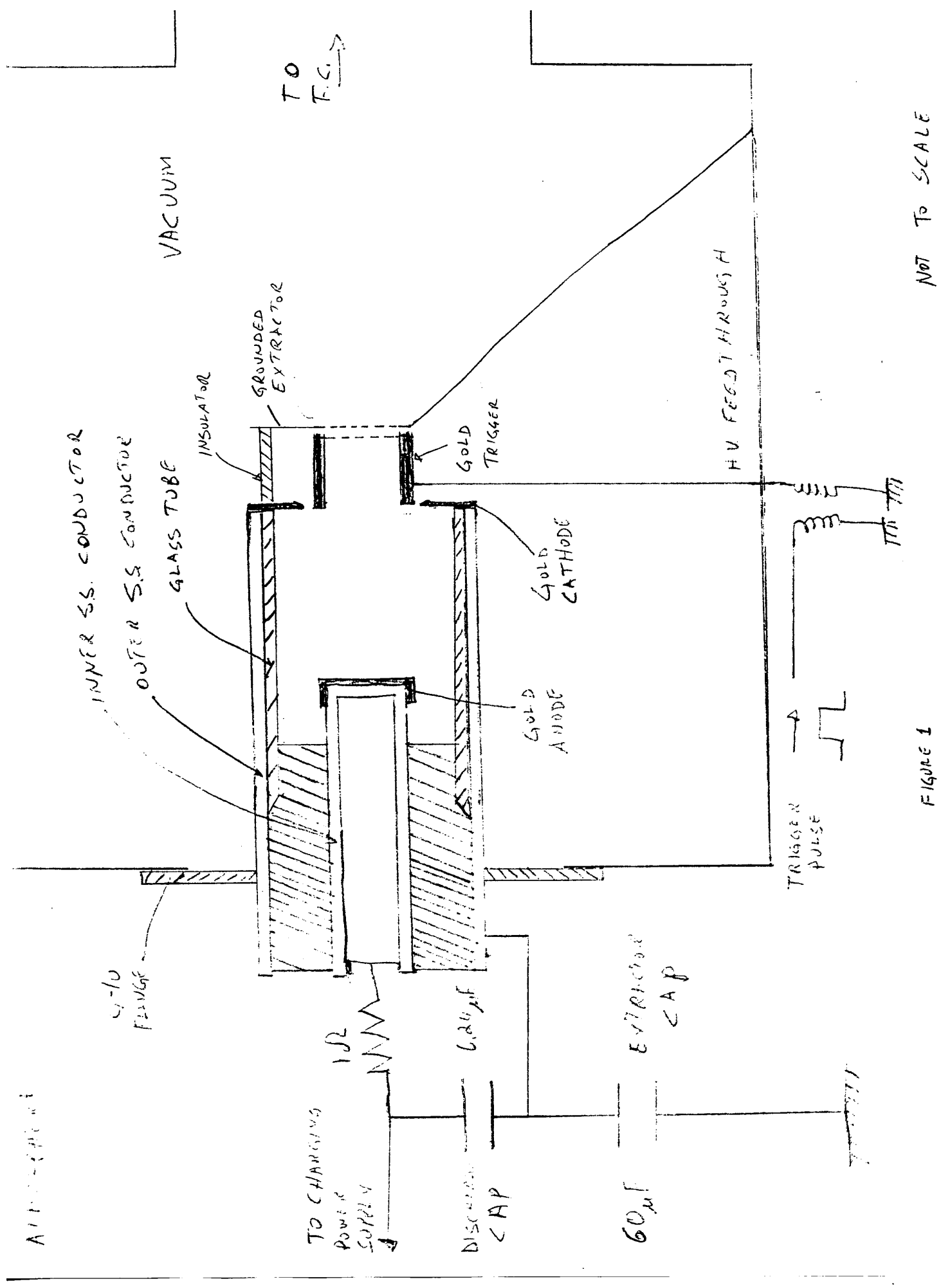




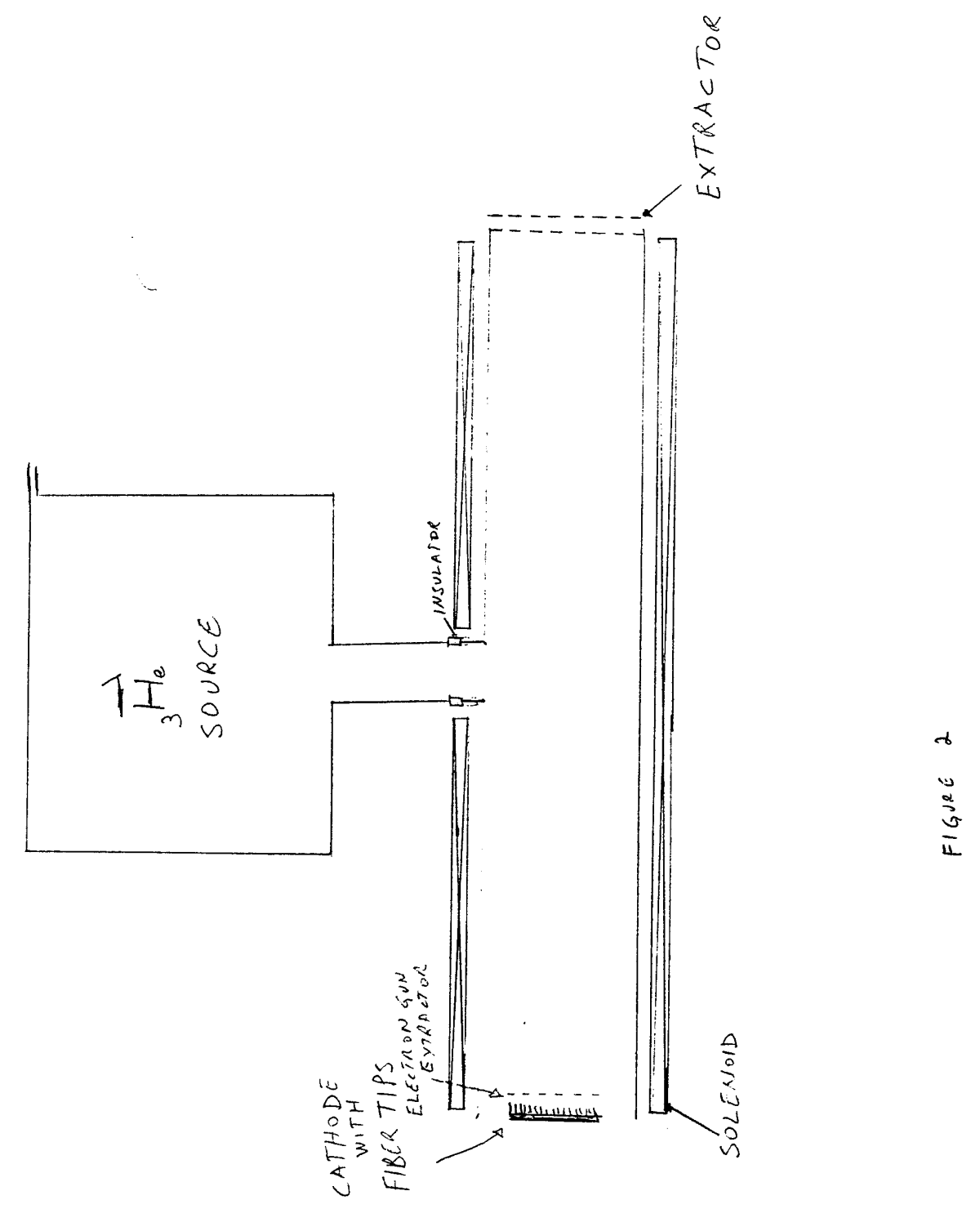

